\title{
Thermo-responsive Polymer Brush Surfaces with Hydrophobic Groups for All-Aqueous Chromatography
}

Kenichi Nagase $^{1}$, Mio Kumazaki ${ }^{1,2}$, Hideko Kanazawa ${ }^{2}$, Jun Kobayashi ${ }^{1}$, Akihiko Kikuchi ${ }^{2}$, Yoshikatsu Akiyama ${ }^{1}$, Masahiko Annaka ${ }^{4}$ and Teruo Okano ${ }^{1} *$

1. Institute of Advanced Biomedical Engineering and Science, Tokyo Women's Medical University, TWIns, 8-1 Kawadacho, Shinjuku, Tokyo 162-8666, Japan.

2. Faculty of Pharmacy, Keio University, 1-5-30 Shibakoen, Minato, Tokyo 105-8512, Japan.

3. Department of Materials Science and Technology, Tokyo University of Science, 2641 Yamazaki, Noda, Chiba 278-8510, Japan

4. Department of Chemistry, Kyushu University, 6-10-1 Hakozaki, Higashiku, Fukuoka 812-8581, Japan CORRESPONDING AUTHOR

*Corresponding author: (Phone) +81-3-5367-9945 Ext. 6201; (Fax) +81-3-3359-6046;

(E-mail) tokano@abmes.twmu.ac.jp

\section{EXPERIMENTAL SECTION}

\section{Retrieving and Analyzing of Grafted Copolymer}

Copolymer grafted silica bead surfaces were treated with concentrated hydrofluoric acid for $3 \mathrm{~h}$, and the solution was neutralized with sodium carbonate. The solution was filtered and dialyzed against Milli-Q water using a dialysis membrane [Spectra/Por standard regenerated cellulose dialysis membrane, Molecular Weight Cut Off (MWCO): 1000] (Spectrum Laboratories, Rancho Dominguez, CA, USA) for 5 days with daily water changed, and the copolymer was recovered by freeze-drying. Numberaverage molecular weights and PDI values of the polymer were determined using a GPC system HLC8320GPC (the columns: TSKgel G3000H and TSKgel G4000H) (Tosoh, Tokyo, Japan). A calibration curve was obtained using poly(ethylene glycol) standards. The flow rate was $1.0 \mathrm{~mL} / \mathrm{min}$. The mobile phase was $N, N$-dimethylformamide $(\mathrm{DMF})$ containing $50 \mathrm{mmol} / \mathrm{L} \mathrm{LiCl}$, and the column temperature was controlled to be $45{ }^{\circ} \mathrm{C}$ using a column oven, and the elution profiles were monitored by a refractometer. 


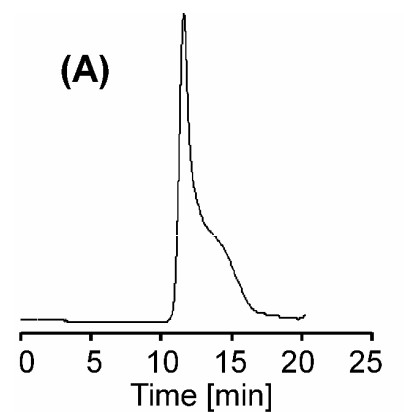

(C)

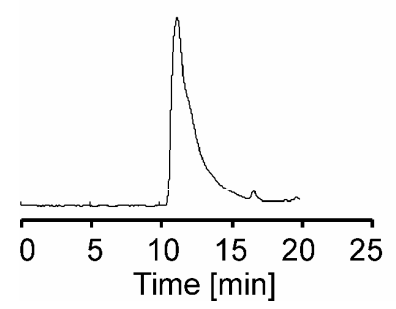

(D)

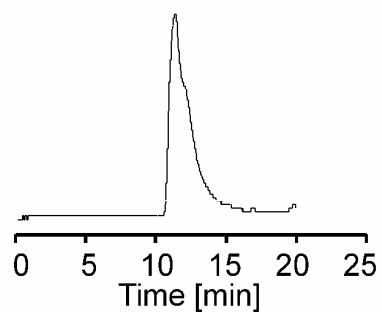

Figure S.1 GPC of the copolymer for obtaining the molecular weight: (A) IPB-0B, (B) IPB-1B, (C) IPB-3B, and (D) IPB-5B (Table 3). Mobile phase is DMF containing $50 \mathrm{mmol} / \mathrm{L} \mathrm{LiCl}$.

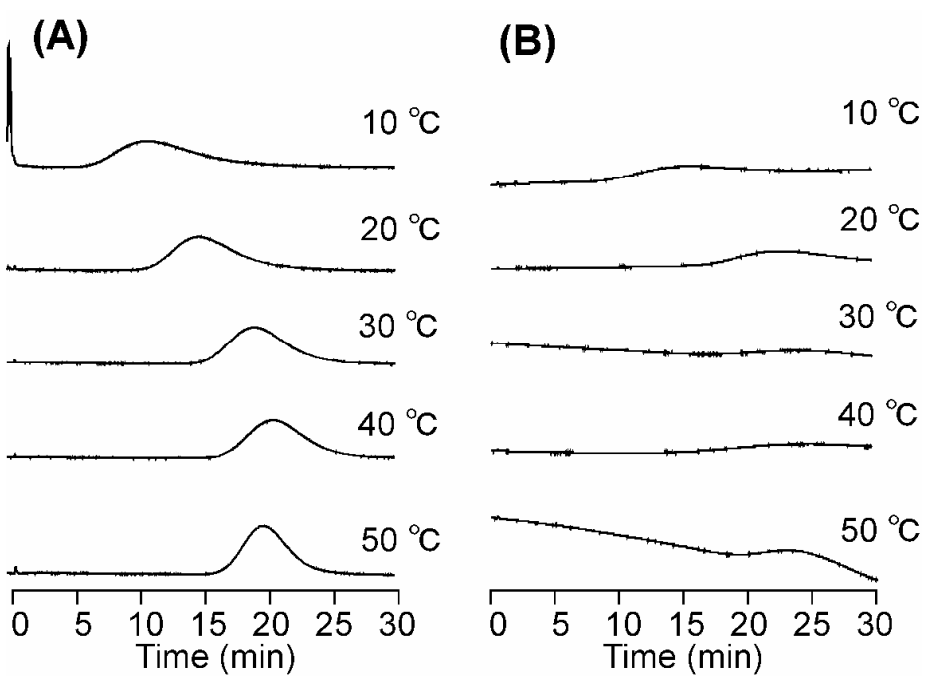

Figure S.2 Chromatograms of dexamethazone on HPLC of which packing materials was poly $(N$-isopropylacrylamideco-butyl methacrylate) grafted silica beads at various temperatures: (A) IPB-1B, and (B) IPB-3B columns (Table 3). The mobile phase is Milli-Q water. Dexamethazone was monitored at $254 \mathrm{~nm}$ by UV detection with a flow rate $1.0 \mathrm{ml} / \mathrm{min}$.
(A)
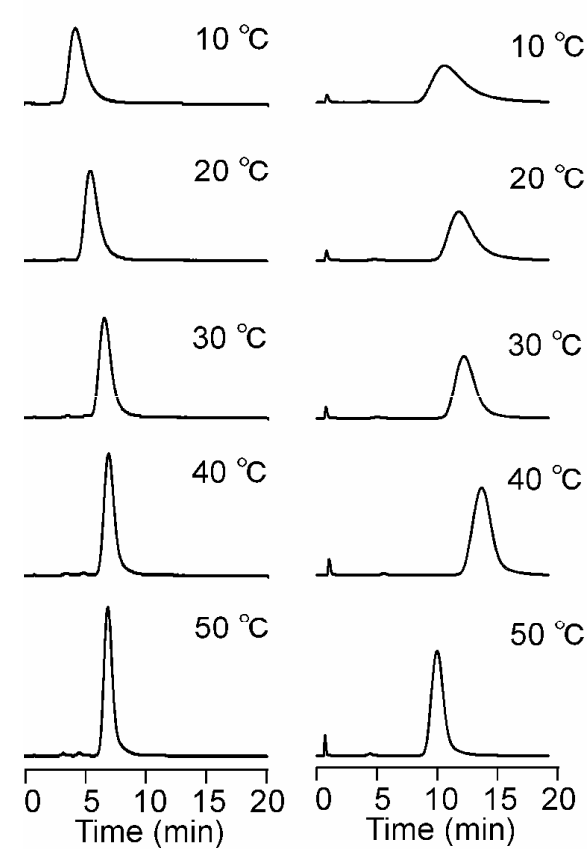

(B)
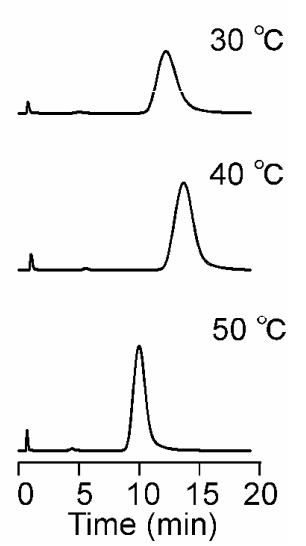

Figure S.3 Chromatograms of ethyl $p$-aminobenzoate on IPB-3B column at various temperatures: (A) using $16.7 \mathrm{mmol} / \mathrm{L}$ phosphate buffer $(\mathrm{pH} 2.5)$, and (B) using $16.7 \mathrm{mmol} / \mathrm{L}$ phosphate buffer $(\mathrm{pH}$ 7.0). Ethyl $p$-aminobenzoate was monitored at $254 \mathrm{~nm}$ by UV detection with a flow rate $1.0 \mathrm{ml} / \mathrm{min}$. 